\title{
Definitions of Empirically Supported Treatment
}

\author{
Robert L. Koegel · Lynn Kern Koegel • \\ Stephen M. Camarata
}

Published online: 11 February 2010

(c) The Author(s) 2010. This article is published with open access at Springerlink.com

\section{Dear Editor:}

We write this letter because we feel the article by Odom et al. (2010a) as well as the erratum by Odom et al. (2010b) would have benefited from the following considerations. An important obstacle for attempts to identify an intervention is the difficulty in distinguishing prepackaged interventions from evolving scientific approaches. An important methodological weakness in such reviews is that they often imply static intervention packages are superior to individualized adaptive approaches, when, in fact, the data supporting the latter are much more plentiful and persuasive. We suggest that such reviews should evaluate the empirical bases for approaches so that relevant supporting evidence is not excluded from the review.

For example, Pivotal Response Treatment (PRT) is an example of a scientifically based approach that has evolved steadily over the years, and is supported by hundreds of programmatic studies (cf. Koegel et al. 2010). However, when one attempts to look at PRT as a static intervention (as if it were complete and final), one is limited to only a select portion of the available research, which could inadvertently mislead the reader with regard to the depth and breadth of the evidence supporting this approach. When one examines the evolving scientific research related to PRT, a different picture emerges. Originally based upon early discrete trial research, primarily the Applied

R. L. Koegel $(\bowtie) \cdot$ L. K. Koegel

University of California, Santa Barbara, CA, USA

e-mail: koegel@education.ucsb.edu

R. L. Koegel · L. K. Koegel

Koegel Autism Consultants, Santa Barbara, CA, USA

S. M. Camarata

Vanderbilt University, Nashville, TN, USA
Behavior Analysis work of Ivar Lovaas and colleagues (cf., Lovaas et al. 1973), a major advance was made in the late 1980s when specific motivational variables, identified through a great many experimental studies, were integrated into an advanced discrete trial approach called the Natural Language Paradigm (Koegel et al. 1987), which was an early forerunner of PRT. The approach continued to be founded upon principles of applied behavior analysis, but incorporated specific motivational variables, that were behaviorally defined, to produce a more rapid, more generalized treatment effect that distinguish PRT from the earlier approach.

Because the approach was implemented in natural environments, rather than in segregated settings, some of the research focused on the naturalistic versus analog dimension of the approach, related studies have been conducted on the rubric of Naturalistic Interventions that extend across goals and settings (e.g. Koegel et al. 1998). Similar research studies found that the approach was producing effects far beyond speech and language, and resulted in direct or collateral improvements in areas such as symbolic play, joint attention, disruptive behavior, social behavior, etc. These research studies, focusing on the broader impact of the approach, called the newest evolution of the approach by the name, Pivotal Response Treatment. By expanding the approach, additional pivotal areas were discovered, extending the Pivotal Response Treatment approach into areas such as responsivity to multiple stimulus input, and child-initiated social interactions. The effect of child-initiated social communication had such a major impact on long term treatment effectiveness, that virtually all recent studies are focusing on, and include child initiations as a key foundational component of Pivotal Response Treatment. Again, for this reason, Pivotal Response Treatment is a scientifically based 
approach that is continually evolving and individualized, not a static intervention, so that these studies supporting evolution of implementation variables should be included in a scientific review.

If one adopts this view for evaluating PRT, more than two hundred scientifically sound articles (according to the guidelines of the American Psychological Association) provide empirical evidence supporting the evolving Pivotal Response Treatment that can be effectively individualized to meet the needs of various family, cultural, child variables along with an adaptation for different settings and target behaviors. Each advance in the PRT approach has produced major improvements in the lives of the children and their families. Yet, we expect that each evolution has the implicit assumption that it will become refined by improved procedures as science produces yet another major advance forward, until 1 day, optimal intervention will be provided to all individuals with autism.

Unfortunately, well intentioned articles, could inadvertently favor static "one size fits all" intervention packages over dynamic, individualized, scientifically developing intervention approaches such as PRT. Although it certainly is important to note that some static interventions do not have any empirical support whatsoever, and thus may not meet even minimal scientific standards, it is potentially misleading to limit the parameters of review so that an approach that has its roots in long-standing scientific findings with hundreds of articles to support it is represented only in a single article. There is no doubt that PRT has its roots in ABA, however, the large data base that has evolved into producing more child-friendly, naturalistic procedures, has significantly advanced the field while continuing to meet the rigorous standards of $\mathrm{ABA}$ and scientific, peer reviewed studies. This is not only true for children with autism, but for a range of disabilities and typical children.

Acknowledgments Research described in this letter was funded by the National Institutes of Health (NIMH and NIDCD), but does not imply the endorsement of NIH.

Open Access This article is distributed under the terms of the Creative Commons Attribution Noncommercial License which permits any noncommercial use, distribution, and reproduction in any medium, provided the original author(s) and source are credited.

\section{References}

Koegel, R. L., O’Dell, M. C., \& Koegel, L. K. (1987). A natural language paradigm for teaching non-verbal autistic children. Journal of Autism and Developmental Disorders, 17, 187-199.

Koegel, R. L., Camarata, S., Koegel, L. K., Ben-Tall, A., \& Smith, A. (1998). Increasing speech intelligibility in children with autism. Journal of Autism and Developmental Disorders, 28, 241-251.

Koegel, R. L., Koegel, L. K., Vernon, T. W., \& Brookman-Frazee, L. I. (2010). Empirically supported pivotal response treatment for children with autism spectrum disorders. In J. Weisz \& A. Kazdin (Eds.), Evidence-based psychotherapies for children and adolescents. New York: Guilford Press.

Lovaas, O. I., Koegel, R. L., Simmons, J. Q., \& Long, J. S. (1973). Some generalization and follow-up measures on autistic children in behavior therapy. Journal of Applied Behavior Analysis, 6, 131-166.

Odom, S. L., Boyd, B. A., Hall, L. J., \& Hume, K. (2010a). Evaluation of comprehensive treatment models for individuals with autism spectrum disorders. Journal of Autism and Developmental Disorders. doi:10.1007/s10803-009-0825-1.

Odom, S. L., Boyd, B. A., Hall, L. J., \& Hume, K. (2010b). Erratum to: Evaluation of comprehensive treatment models for individuals with autism spectrum disorders. Journal of Autism and Developmental Disorders. doi:10.1007/s10803-009-0873-6. 